\title{
Influence of Graded Doses of Organic and Inorganic Nutrients on Growth, Yield and Economics of Tikhur (Curcuma aungustifolia Roxb.) in Inceptisol of Bastar Plateau
}

\author{
Karuna Kant Joshi*, Girijesh Kumar Sharma, Tejpal Chandrakar and Deo Shankar \\ Section of Soil Science and Agricultural Chemistry, Indira Gandhi Krishi Vishwavidyalaya, \\ Shaheed Gundadhoor College of Agriculture and Research Station, \\ Jagdalpur-494005, Chhattisgarh, India \\ *Corresponding author
}

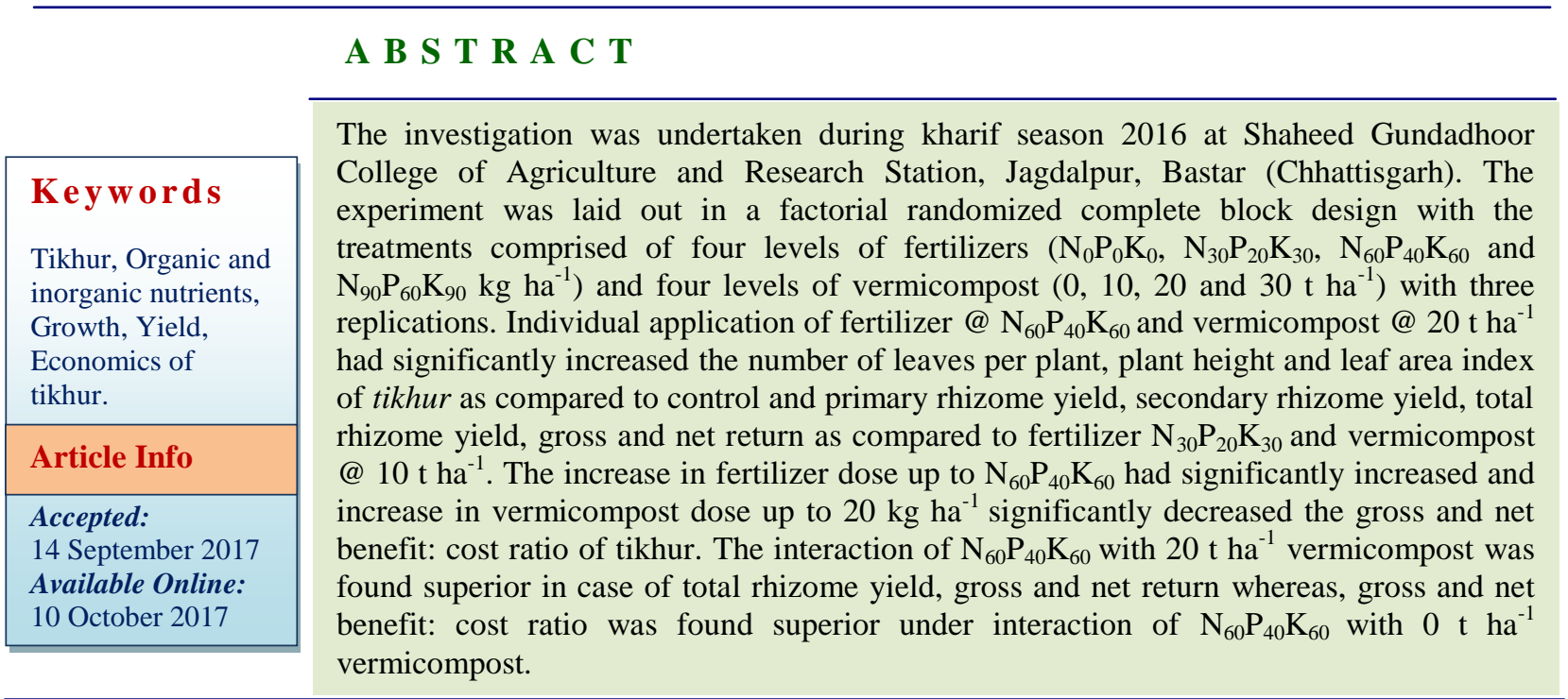

\section{Introduction}

Tikhur (Curcuma angustifolia; family Zingiberaceae) is a rhizomatous herb also known as white turmeric or East Indian arrowroot. It is generally propagated by rhizomes and a good source of starch and fibre (Misra and Dixit, 1983). The plant grows wild in many places, it is found in moist and cool situations at altitudes of about $450 \mathrm{~m}$. Tikhur cultivated as medicinal crop in many parts of the state under moist deciduous mixed and Sal forest of Madhya Pradesh, Chhattisgarh and Jharkhand. Its cultivation has now been undertaken by the farmers of Bastar on a large area. Total area of tuber crops in Chhattisgarh is 2391.13 ha, total production is $64880.25 \mathrm{t}$ and average productivity of all tuber crops is $22.70 \mathrm{t} \mathrm{ha}^{-1}$ as per the data recorded though conventional survey from all 27 districts of Chhattisgarh during the year 2013-14.

Farmer's yields are less due to lack of improved and high starch yielding genotypes and proper nutrient management practices. It 
is understood that there is a wide gap between potential yield and the yield obtained under actual field situations for tuber crops. Among the different factors contributing to this yield gap, soil-plant nutrition is worthy of mention as soil fertility management and proper nutrition of these crops can result in large yield gains. It is well known that addition of organic manures has shown considerable increase in crop yield, quality and exert significant influence on physical, chemical and biological properties of soil. Work related to nutrient management in Indian arrowroot was carried out by some workers. Ramesan et al., (1996) studied the nutritional requirement of arrowroot on biomass production, rhizome yield, nutrient uptake and available $\mathrm{N}, \mathrm{P}$ and $\mathrm{K}$ status of the soil and found that $\mathrm{N}$ and $\mathrm{K} @$ 50 and $75 \mathrm{~kg} \mathrm{ha}^{-1}$ significantly increased rhizome yield. Maheswarappa et al., (2000) and Veena (2000) reported highest uptake of $\mathrm{N}$ and $\mathrm{K}$ at the highest level of fertilization in arrowroot intercropped in coconut gardens. Suja et al., (2006) studied the influence of nutrient management on arrowroot yield, nutrient uptake and soil nutrient status and found that application of N P and K @ $50: 25: 75 \mathrm{~kg} \mathrm{ha}^{-1}$ was ideal to obtain better yield (23.29 $\left.\mathrm{t} \mathrm{ha}^{-1}\right)$, higher uptake of nutrients and substantial improvement in the nutrient status. Looking to very limited information on the proper nutrient doses to maximize yield of Indian arrowroot, present investigation was undertaken with the objective to investigate the influence of graded levels of organic and inorganic sources of nutrients on growth, yield and economics of Tikhur (Curcuma aungustifolia Roxb.) in Inceptisol of Bastar Plateau region of Chhattisgarh

\section{Materials and Methods}

The investigation was undertaken during the kharif season of 2016 at Indira Gandhi Krishi Vishwavidyalaya, Shaheed Gundadhoor College of Agriculture and Research Station, Jagdalpur, Bastar (Chhattisgarh). The experiment was laid out in a factorial randomized complete block design with the treatments comprised of four levels of fertilizers $\left(\mathrm{N}_{0} \mathrm{P}_{0} \mathrm{~K}_{0}, \mathrm{~N}_{30} \mathrm{P}_{20} \mathrm{~K}_{30}, \mathrm{~N}_{60} \mathrm{P}_{40} \mathrm{~K}_{60}\right.$ and $\left.\mathrm{N}_{90} \mathrm{P}_{60} \mathrm{~K}_{90} \mathrm{~kg} \mathrm{ha}{ }^{-1}\right)$ and four levels of vermicompost $\left(0,10,20\right.$ and $\left.30 \mathrm{t} \mathrm{ha}^{-1}\right)$ and three replications.

The experimental soil was loamy in texture, comes under Inceptisols order and had initial soil physicochemical properties like $46 \%$ sand, $30 \%$ silt, $24 \%$ clay, $5.5 \mathrm{pH}, 0.03 \mathrm{dSm}^{-1}$ electrical conductivity, $0.71 \%$ organic carbon, $1.43 \mathrm{~g} \mathrm{~cm}^{-3}$ bulk density, $14.5 \mathrm{C} \mathrm{mol}(\mathrm{p}+) \mathrm{kg}^{-1}$ cation exchange capacity, $202.2 \mathrm{~kg} \mathrm{\textrm {ha } ^ { - 1 }}$ available $\mathrm{N}, 13.9 \mathrm{~kg} \mathrm{ha}^{-1}$ available $\mathrm{P}, 206 \mathrm{~kg}$ $\mathrm{ha}^{-1}$ available $\mathrm{K}, 16.6 \mathrm{~kg} \mathrm{ha}^{-1}$ available $\mathrm{S}$, $2.51 \mathrm{meq} / 100 \mathrm{~g}$ available $\mathrm{Ca}, 1.82 \mathrm{meq} / 100 \mathrm{~g}$ soil available $\mathrm{Mg}$. The nutritional composition of vermicompost used in the study was $1.4 \% \mathrm{~N}, 0.6 \% \mathrm{P}$ and $1.1 \% \mathrm{~K}$. The planting of Tikhur was done on May 17, 2016. Full dose of vermicompost, phosphorus and potassium and $1 / 3$ dose of nitrogen, as per treatment, were applied in the form of diammonium phosphate, muriate of potash and urea, at the time of planting and there maining dose of nitrogen was applied at 60 and 75 days after planting. The Intercultural operation like, hand weeding done three times at 60,90 and 120 days after planting respectively, earthing up done at 110 days after planting, spraying of macoban (carbendazim + mancozeb) fungicide for the control of blight disease at 100 days after sowing and crops are grown in rainfed field conditions. The crop was harvested at 30 December 2016 after complete maturity, as indicated by the leaf drying and falling down of plants.

For recording observations, randomly ten plants were selected in each of the plot. The Plant height was recorded with the help of a meter scale from ground level to upper most point of plant, total number of leaves recorded which included all the leaves from the base of 
the plants, leaf area index was estimated from randomly plants having 3 functional leaves and estimated by dividing the total leaf area of the plant by ground area, dry matter of rhizomes $(\%)$ was calculated by dividing the dry weight of rhizomes by fresh weight of rhizome multiplied by hundred, After harvesting of whole rhizomes, primary rhizomes (fingers) were separated from mother rhizomes of selected plants and weighted and averaged for primary rhizome weight per plant, After harvesting of whole rhizomes, secondary rhizomes (fingers) were separated from primary rhizomes of selected plants and weighted and averaged for secondary rhizome and total weight of rhizomes harvested from selected plants was recorded during maturity. The gross return was obtained by the using local market price of tikhur powder @ 500 Rs. $\mathrm{kg}^{-1}$ calculated by multiplied starch yield to local market price, net return estimated by gross return minus cost of cultivation, gross benefit: cost ratio calculated by dividing gross return by cost of cultivation and net benefit: cost ratio calculated by dividing net return by cost of cultivation.

\section{Results and Discussion}

\section{Number of leaves per plant}

The data presented in (Table 1) revealed that organic and inorganic nutrients had significant effect on number of leaves per plant. However, the interaction effect of fertilizers with vermicompost on number of leaves per plant was not found significant. The number of leaves per plant of tikhur ranges from 8.70 to 9.16 due to vermicompost levels with the highest value under application of vermicompost @ $30 \mathrm{t} \mathrm{ha}^{-1}$ which was significantly higher than 0 and $10 \mathrm{t}$ $\mathrm{ha}^{-1}$ but at par with $20 \mathrm{t} \mathrm{ha}^{-1}$. The number of leaves per plant of tikhur ranges from 8.73 to 9.10 due to fertilizers levels with the highest value under application of fertilizer @
$\mathrm{N}_{90} \mathrm{P}_{60} \mathrm{~K}_{90} \mathrm{~kg} \mathrm{ha}^{-1}$ which was significantly higher than $\mathrm{N}_{0} \mathrm{P}_{0} \mathrm{~K}_{0}$ and $\mathrm{N}_{30} \mathrm{P}_{20} \mathrm{~K}_{30}$ but at par with $\mathrm{N}_{60} \mathrm{P}_{40} \mathrm{~K}_{60}$. Similar findings were also reported by Odedina et al., (2012) and Thomas et al., (2002).

\section{Plant height}

Plant height of tikhur was influenced significantly (Table 1) with individual levels of organic and inorganic nutrients, however, their interaction was not found significant. The plant height of tikhur ranges from 42.5 to $48.6 \mathrm{~cm}$ due to fertilizers levels with the highest value under application of fertilizer @ $\mathrm{N}_{90} \mathrm{P}_{60} \mathrm{~K}_{90} \mathrm{~kg}$ ha $^{-1}$ which was significantly higher than $\mathrm{N}_{0} \mathrm{P}_{0} \mathrm{~K}_{0}$ and $\mathrm{N}_{30} \mathrm{P}_{20} \mathrm{~K}_{30}$ but at par with $\mathrm{N}_{60} \mathrm{P}_{40} \mathrm{~K}_{60}$. The plant height of tikhur ranges from 42.1 to $48.7 \mathrm{~cm}$ due to vermicompost levels with the highest value under application of vermicompost @ 30 t ha

${ }^{1}$ which was significantly higher than 0 and 10 $\mathrm{t} \mathrm{ha}^{-1}$ but at par with $20 \mathrm{t} \mathrm{ha}^{-1}$. Similar findings were reported by many workers (Iwuagwu et al., 2016 and Mama et al., 2016).

\section{Leaf area index}

The organic and inorganic nutrients, individually had significant effect on leaf area index of tikhur (Table 1), however, their interaction was not found significant. The leaf area index ranges from 0.93 to 1.23 and 0.90 to 1.24 with the highest under application of fertilizer @ $\mathrm{N}_{90} \mathrm{P}_{60} \mathrm{~K}_{90} \mathrm{~kg} \quad \mathrm{ha}^{-1}$ and vermicompost @ 30t ha ${ }^{-1}$, respectively. The effect of fertilizer @ $\mathrm{N}_{90} \mathrm{P}_{60} \mathrm{~K}_{90} \mathrm{kgha}^{-1}$ was significantly higher than $\mathrm{N}_{0} \mathrm{P}_{0} \mathrm{~K}_{0}$ and $\mathrm{N}_{30} \mathrm{P}_{20} \mathrm{~K}_{30}$ but at par with $\mathrm{N}_{60} \mathrm{P}_{40} \mathrm{~K}_{60}$, similarly vermicompost @30t ha ${ }^{-1}$ had significantly increased leaf area index as compared to 0 and $10 \mathrm{t} \mathrm{ha}^{-1}$ but its effect was at par as compared to vermicompost dose @ 20t ha ${ }^{-1}$. Similar findings were also recorded by Swadija et al., (2013) and Sandhu et al., (2014). 


\section{Dry matter percent of rhizome}

The dry matter percent of rhizome (Table 1) was significantly influenced by individual levels of organic and inorganic nutrients, however, their interaction was not found significant. The dry matter percent of rhizome ranges from 30.3 to $33.9 \%$ and 29.9 to $34.1 \%$ with the highest value under application of fertilizer @ $\mathrm{N}_{90} \mathrm{P}_{60} \mathrm{~K}_{90} \mathrm{~kg} \quad \mathrm{ha}^{-1}$ and vermicompost @ 30t ha ${ }^{-1}$, respectively. Increasing fertilizer level up to $\mathrm{N}_{60} \mathrm{P}_{40} \mathrm{~K}_{60} \mathrm{~kg}$ $\mathrm{ha}^{-1}$ and vermicompost up to $20 \mathrm{t} \mathrm{ha}^{-1}$ significantly increased dry matter percent of rhizome. Similar findings were also recorded by several workers (Amanullah et al., 2007, Ogbomo and Remison, 2008, Verma et al., 2012, Ezeocha et al., 2014 and Hota et al., 2014).

\section{Primary rhizome yield}

The primary rhizome yield was significantly influenced by individual levels of organic and inorganic nutrients (Table 1), however, their interaction was not found significant.

The primary rhizome yield ranges from 29.5 to $40.4 \mathrm{q} \mathrm{ha}^{-1}$ and 30.2 to $39.9 \mathrm{q} \mathrm{ha}^{-1}$ with the highest value under application of fertilizer @ $\mathrm{N}_{90} \mathrm{P}_{60} \mathrm{~K}_{90} \mathrm{~kg} \mathrm{ha}^{-1}$ and vermicompost @ 30t $\mathrm{ha}^{-1}$, respectively. The effect of fertilizer @ $\mathrm{N}_{90} \mathrm{P}_{60} \mathrm{~K}_{90} \mathrm{~kg} \mathrm{ha}{ }^{-1}$ was significantly higher than $\mathrm{N}_{0} \mathrm{P}_{0} \mathrm{~K}_{0}$ and $\mathrm{N}_{30} \mathrm{P}_{20} \mathrm{~K}_{30}$ but at par with $\mathrm{N}_{60} \mathrm{P}_{40} \mathrm{~K}_{60} \mathrm{~kg} \mathrm{ha}^{-1}$, similarly vermicompost @ $30 \mathrm{tha}^{-1}$ had significantly increased primary rhizome yield as compared to 0 and $10 \mathrm{tha}^{-1}$ but its effect was at par as compared to vermicompost dose @ 20t ha ${ }^{-1}$.

\section{Secondary rhizome yield}

The organic and inorganic nutrients had significant effect on secondary rhizome yield (Table 1), however, their interaction was not found significant. The secondary rhizome yield ranges from 13.4 to $18.9 \mathrm{q} \mathrm{ha}^{-1}$ and 13.3 to $18.6 \mathrm{q} \mathrm{ha}^{-1}$ with the highest value under application of fertilizer @ $\mathrm{N}_{90} \mathrm{P}_{60} \mathrm{~K}_{90} \mathrm{~kg} \mathrm{ha}^{-1}$ and vermicompost @30t ha ${ }^{-1}$, respectively. The effect of fertilizer @ $\mathrm{N}_{90} \mathrm{P}_{60} \mathrm{~K}_{90} \mathrm{~kg} \mathrm{ha}^{-1}$ was significantly higher than $\mathrm{N}_{0} \mathrm{P}_{0} \mathrm{~K}_{0}$ and $\mathrm{N}_{30} \mathrm{P}_{20} \mathrm{~K}_{30}$ but at par with $\mathrm{N}_{60} \mathrm{P}_{40} \mathrm{~K}_{60} \mathrm{~kg} \mathrm{ha}^{-1}$, similarly vermicompost @ 30t ha ${ }^{-1}$ had significantly increased secondary rhizome yield as compared to 0 and $10 \mathrm{tha}^{-1}$ but its effect was at par as compared to vermicompost dose @ 20t ha ${ }^{-1}$.

\section{Total rhizome yield}

The data presented in (Tables 1 and 2) revealed that organic and inorganic nutrients had significant simple and interaction effect on total rhizome yield. The total rhizome yield ranges from 62.4 to $104.9 \mathrm{q} \mathrm{ha}^{-1}$ to 75.7 to $97.8 \mathrm{q} \mathrm{ha}^{-1}$ with the highest value under application of fertilizer @ $\mathrm{N}_{90} \mathrm{P}_{60} \mathrm{~K}_{90} \mathrm{~kg} \mathrm{ha}^{-1}$ and vermicompost @30t ha ${ }^{-1}$, respectively.

In case of interaction effect (Table 2), the total rhizome yield of tikhur increased under all the fertilizer levels as we increased vermicompost levels up to $20 \mathrm{t} \mathrm{ha}^{-1}$, increasing vermicompost levels, their after, did not increase total rhizome yield significantly. Similarly, the total rhizome yield of tikhur increased under all the vermicompost levels as we increased fertilizer levels up to $\mathrm{N}_{60} \mathrm{P}_{40} \mathrm{~K}_{60}$, increasing fertilizer level further did not statistically increases the total rhizome yield.

The higher total rhizome yield $110.4 \mathrm{q} \mathrm{ha}^{-1}$ was achieved due to $30 \mathrm{t} \mathrm{ha}^{-1}$ vermicompost $\mathrm{X}$ $\mathrm{N}_{90} \mathrm{P}_{60} \mathrm{~K}_{90} \mathrm{~kg} \mathrm{ha}^{-1}$ which was statistically at par with $\mathrm{N}_{60} \mathrm{P}_{40} \mathrm{~K}_{60}$ X 30 t ha $^{-1}$ vermicompost, $\mathrm{N}_{90} \mathrm{P}_{60} \mathrm{~K}_{90} \quad \mathrm{X} 20 \mathrm{t} \mathrm{ha}^{-1}$ vermicompost and $\mathrm{N}_{60} \mathrm{P}_{40} \mathrm{~K}_{60}$ X $20 \mathrm{t} \mathrm{ha}^{-1}$ vermicompost. The combination of $\mathrm{N}_{60} \mathrm{P}_{40} \mathrm{~K}_{60} \quad \mathrm{X} \quad 20 \quad \mathrm{t} \quad \mathrm{ha}^{-1}$ vermicompost was found superior as it saves cost on fertilizer and vermicompost and provides yield comparable to other higher doses of fertilizer and vermicompost. 
Table.1 Effect of graded doses of inorganic and organic sources of nutrients on growth, yield and economics of Tikhur

\begin{tabular}{|c|c|c|c|c|c|c|c|c|c|c|c|}
\hline Treatments & $\begin{array}{c}\text { No of } \\
\text { leaves per } \\
\text { plant }\end{array}$ & $\begin{array}{l}\text { Plant } \\
\text { height } \\
(\mathrm{cm})\end{array}$ & LAI & $\begin{array}{c}\text { DMCR } \\
(\%)\end{array}$ & $\begin{array}{c}\text { PRY } \\
\left(\mathrm{q} \mathrm{ha}^{-1}\right)\end{array}$ & $\begin{array}{c}\text { SRY } \\
\left(\mathrm{q} \mathrm{ha}^{-1}\right)\end{array}$ & $\begin{array}{c}\text { TRY } \\
\left(\mathrm{q} \mathrm{ha}^{-1}\right)\end{array}$ & $\begin{array}{c}\text { Gross } \\
\text { Return } \\
\left(\text { Rs. ha }{ }^{-1}\right)\end{array}$ & $\begin{array}{c}\text { Net } \\
\text { Return } \\
\left(\text { Rs.ha }^{-1}\right)\end{array}$ & $\begin{array}{c}\text { Gross } \\
\text { B:C ratio } \\
\left(\text { Rs. } \text { Re. }^{-1}\right)\end{array}$ & $\begin{array}{c}\text { Net B:C } \\
\text { ratio } \\
\left(\text { Rs.Re }^{-1}\right)\end{array}$ \\
\hline \multicolumn{12}{|c|}{ Fertilizer Levels $\left(\mathrm{kg} \mathrm{ha}^{-1}\right)$} \\
\hline $\mathrm{N}_{0} \mathrm{P}_{0} \mathrm{~K}_{0}$ & $8.73^{\mathrm{a}}$ & $42.5^{\mathrm{a}}$ & $0.93^{\mathrm{a}}$ & $30.3^{\mathrm{a}}$ & $29.5^{\mathrm{a}}$ & $13.4^{\mathrm{a}}$ & $62.4^{\mathrm{a}}$ & $307444^{\mathrm{a}}$ & $158485^{\mathrm{a}}$ & $2.13^{\mathrm{a}}$ & $1.14^{\mathrm{a}}$ \\
\hline $\mathrm{N}_{30} \mathrm{P}_{20} \mathrm{~K}_{30}$ & $8.88^{\mathrm{ab}}$ & $44.4^{\mathrm{ab}}$ & $1.02^{\mathrm{ab}}$ & $31.2^{\mathrm{a}}$ & $33.3^{\mathrm{b}}$ & $15.0^{\mathrm{b}}$ & $85.8^{\mathrm{b}}$ & $440034^{\mathrm{b}}$ & $262137^{\mathrm{b}}$ & $2.55^{\mathrm{b}}$ & $1.55^{\mathrm{b}}$ \\
\hline $\mathrm{N}_{60} \mathrm{P}_{40} \mathrm{~K}_{60}$ & $8.97^{\mathrm{b}}$ & $45.2^{\mathrm{b}}$ & $1.09^{\mathrm{b}}$ & $32.4^{\mathrm{ab}}$ & $38.3^{\mathrm{c}}$ & $18.2^{\mathrm{c}}$ & $104.0^{\mathrm{c}}$ & $580498^{c}$ & $372090^{c}$ & $2.86^{\mathrm{c}}$ & $1.86^{\mathrm{c}}$ \\
\hline $\mathrm{N}_{90} \mathrm{P}_{60} \mathrm{~K}_{90}$ & $9.10^{\mathrm{b}}$ & $48.6^{\mathrm{c}}$ & $1.23^{\mathrm{b}}$ & $33.9^{\mathrm{b}}$ & $40.4^{\mathrm{c}}$ & $18.9^{\mathrm{c}}$ & $104.9^{\mathrm{c}}$ & $587532^{c}$ & $375110^{c}$ & $2.84^{\mathrm{c}}$ & $1.84^{\mathrm{c}}$ \\
\hline $\mathrm{CD}(\mathrm{P}=0.05)$ & 0.23 & 2.6 & 0.15 & 2.2 & 2.1 & 0.8 & 1.1 & 7929 & 6344 & 0.03 & 0.03 \\
\hline \multicolumn{12}{|c|}{ Vermicompost Levels $\left(\mathrm{t} \mathrm{ha}^{-1}\right)$} \\
\hline 0 & $8.70^{\mathrm{a}}$ & $42.1^{\mathrm{a}}$ & $0.90^{\mathrm{a}}$ & $29.9^{\mathrm{a}}$ & $30.2^{\mathrm{a}}$ & $13.3^{\mathrm{a}}$ & $75.7^{\mathrm{a}}$ & $382903^{\mathrm{a}}$ & $260176^{\mathrm{a}}$ & $3.04^{\mathrm{a}}$ & $2.04^{\mathrm{a}}$ \\
\hline 10 & $8.84^{\mathrm{ab}}$ & $43.7^{\mathrm{ab}}$ & $1.00^{\mathrm{ab}}$ & $31.1^{\mathrm{ab}}$ & $33.5^{\mathrm{b}}$ & $15.7^{\mathrm{b}}$ & $86.8^{\mathrm{b}}$ & $458264^{b}$ & $290466^{\mathrm{b}}$ & $2.68^{b}$ & $1.68^{\mathrm{b}}$ \\
\hline 20 & $8.99^{b}$ & $46.1^{b}$ & $1.13^{b}$ & $32.7^{\mathrm{b}}$ & $37.9^{c}$ & $17.9^{\mathrm{c}}$ & $96.7^{\mathrm{c}}$ & $533462^{c}$ & $320623^{c}$ & $2.48^{\mathrm{c}}$ & $1.48^{\mathrm{c}}$ \\
\hline 30 & $9.16^{\mathrm{b}}$ & $48.7^{\mathrm{b}}$ & $1.24^{\mathrm{b}}$ & $34.1^{\mathrm{b}}$ & $39.9^{c}$ & $18.6^{\mathrm{c}}$ & $97.8^{\mathrm{c}}$ & $540879^{c}$ & $296557^{b}$ & $2.19^{c}$ & $1.19^{c}$ \\
\hline $\mathrm{CD}(\mathrm{P}=0.05)$ & 0.23 & 2.6 & 0.15 & 2.2 & 2.1 & 0.8 & 1.1 & 7929 & 6344 & 0.03 & 0.03 \\
\hline $\begin{array}{l}\text { Interaction } \\
\mathbf{C D}(\mathbf{P}=0.05)\end{array}$ & N.S. & N.S. & N.S. & N.S. & N.S. & N.S. & 2.14 & 15859 & 12687 & 0.05 & 0.51 \\
\hline
\end{tabular}

Where, LAI= Leaf area index, DMCR= Dry matter content of rhizome (\%), PRY= Primary rhizome yield, SRY= Secondary rhizome yield and TRY=Total rhizome yield. Same small letter in a column have no significant difference as per Duncan's Multiple Range Test. 
Table.2 Interaction effects of graded doses of inorganic and organic sources of nutrients on yield and economic parameters of Tikhur

\begin{tabular}{|c|c|c|c|c|c|}
\hline \multirow{2}{*}{$\begin{array}{l}\text { Fertilizer levels } \\
\left(\mathrm{kg} \mathrm{ha}^{-1}\right)\end{array}$} & \multicolumn{4}{|c|}{ Vermicompost levels $\left(\mathrm{t} \mathrm{ha}^{-1}\right)$} & \multirow{2}{*}{$\begin{array}{l}\mathrm{CD}(\mathrm{P}=0.05) \\
\text { for Interaction }\end{array}$} \\
\hline & $\mathbf{0}$ & 10 & 20 & 30 & \\
\hline \multicolumn{6}{|c|}{ Total Rhizome Yield $\left(\mathrm{q} \mathrm{ha}^{-1}\right)$} \\
\hline $\mathrm{N}_{0} \mathrm{P}_{0} \mathrm{~K}_{0}$ & $44.6^{\mathrm{aA}}$ & $57.9^{\mathrm{aB}}$ & $72.8^{\mathrm{aC}}$ & $74.1^{\mathrm{aC}}$ & \multirow{4}{*}{2.1} \\
\hline $\mathrm{N}_{30} \mathrm{P}_{20} \mathrm{~K}_{30}$ & $68.0^{\mathrm{bA}}$ & $82.9^{\mathrm{bB}}$ & $95.5^{\mathrm{bC}}$ & $96.6^{\mathrm{bC}}$ & \\
\hline $\mathrm{N}_{60} \mathrm{P}_{40} \mathrm{~K}_{60}$ & $94.2^{\mathrm{cA}}$ & $102.5^{\mathrm{cB}}$ & $109.2^{\mathrm{cC}}$ & $110.0^{\mathrm{cC}}$ & \\
\hline $\mathrm{N}_{90} \mathrm{P}_{60} \mathrm{~K}_{90}$ & $95.9^{\mathrm{cA}}$ & $104.0^{\mathrm{cB}}$ & $109.3^{\mathrm{cC}}$ & $110.4^{\mathrm{cC}}$ & \\
\hline \multicolumn{6}{|c|}{ Gross Return (Rs. ha $\left.{ }^{-1}\right)$} \\
\hline $\mathrm{N}_{0} \mathrm{P}_{0} \mathrm{~K}_{0}$ & $209230^{\mathrm{aA}}$ & $279586^{\mathrm{aB}}$ & $365777^{\mathrm{aC}}$ & $375180^{\mathrm{aC}}$ & \multirow{4}{*}{15859} \\
\hline $\mathrm{N}_{30} \mathrm{P}_{20} \mathrm{~K}_{30}$ & $326228^{\mathrm{bA}}$ & $417906^{\mathrm{bB}}$ & $503759^{\mathrm{bC}}$ & $512244^{\mathrm{bC}}$ & \\
\hline $\mathrm{N}_{60} \mathrm{P}_{40} \mathrm{~K}_{60}$ & $489976^{\mathrm{cA}}$ & $563941^{\mathrm{cB}}$ & $631425^{\mathrm{cC}}$ & $636649^{\mathrm{cC}}$ & \\
\hline $\mathrm{N}_{90} \mathrm{P}_{60} \mathrm{~K}_{90}$ & $506176^{\mathrm{dA}}$ & $571625^{\mathrm{cB}}$ & $632885^{\mathrm{cC}}$ & $639443^{\mathrm{cC}}$ & \\
\hline \multicolumn{6}{|c|}{ Net Return (Rs. ha $\left.{ }^{-1}\right)$} \\
\hline $\mathrm{N}_{0} \mathrm{P}_{0} \mathrm{~K}_{0}$ & $124914^{\mathrm{aA}}$ & $151199^{\mathrm{aB}}$ & $190152^{\mathrm{aC}}$ & $167674^{\mathrm{aC}}$ & \multirow{4}{*}{12687} \\
\hline $\mathrm{N}_{30} \mathrm{P}_{20} \mathrm{~K}_{30}$ & $216092^{\mathrm{bA}}$ & $259435^{\mathrm{bB}}$ & $298117^{\mathrm{bC}}$ & $274905^{\mathrm{bC}}$ & \\
\hline $\mathrm{N}_{60} \mathrm{P}_{40} \mathrm{~K}_{60}$ & $344673^{\mathrm{cA}}$ & $373845^{\mathrm{cB}}$ & $397831^{\mathrm{cC}}$ & $372012^{\mathrm{cC}}$ & \\
\hline $\mathrm{N}_{90} \mathrm{P}_{60} \mathrm{~K}_{90}$ & $355025^{\mathrm{cA}}$ & $377383^{\mathrm{cB}}$ & $396392^{\mathrm{cC}}$ & $371639^{\mathrm{cC}}$ & \\
\hline \multicolumn{6}{|c|}{ Gross Benefit: Cost ratio (Rs. $\left.\operatorname{Re}^{-1}\right)$} \\
\hline $\mathrm{N}_{0} \mathrm{P}_{0} \mathrm{~K}_{0}$ & $2.48^{\mathrm{aA}}$ & $2.18^{\mathrm{aB}}$ & $2.08^{\mathrm{aC}}$ & $1.81^{\mathrm{aD}}$ & \multirow{4}{*}{0.05} \\
\hline $\mathrm{N}_{30} \mathrm{P}_{20} \mathrm{~K}_{30}$ & $2.96^{\mathrm{bA}}$ & $2.64^{\mathrm{bB}}$ & $2.45^{\mathrm{bC}}$ & $2.16^{\mathrm{bD}}$ & \\
\hline $\mathrm{N}_{60} \mathrm{P}_{40} \mathrm{~K}_{60}$ & $3.37^{\mathrm{cA}}$ & $2.97^{\mathrm{cB}}$ & $2.70^{\mathrm{cC}}$ & $2.41^{\mathrm{cD}}$ & \\
\hline $\mathrm{N}_{90} \mathrm{P}_{60} \mathrm{~K}_{90}$ & $3.35^{\mathrm{cA}}$ & $2.94^{\mathrm{cB}}$ & $2.67^{\mathrm{cC}}$ & $2.39^{\mathrm{cD}}$ & \\
\hline \multicolumn{6}{|c|}{ Net Benefit: Cost ratio (Rs. Re. $^{-1}$ ) } \\
\hline $\mathrm{N}_{0} \mathrm{P}_{0} \mathrm{~K}_{0}$ & $1.48^{\mathrm{aA}}$ & $1.18^{\mathrm{aB}}$ & $1.08^{\mathrm{aC}}$ & $0.81^{\mathrm{aD}}$ & \multirow{4}{*}{0.05} \\
\hline $\mathrm{N}_{30} \mathrm{P}_{20} \mathrm{~K}_{30}$ & $1.96^{\mathrm{bA}}$ & $1.64^{\mathrm{bB}}$ & $1.45^{\mathrm{bC}}$ & $1.16^{\mathrm{bD}}$ & \\
\hline $\mathrm{N}_{60} \mathrm{P}_{40} \mathrm{~K}_{60}$ & $2.37^{\mathrm{cA}}$ & $1.97^{\mathrm{cB}}$ & $1.70^{\mathrm{cC}}$ & $1.41^{\mathrm{cD}}$ & \\
\hline $\mathrm{N}_{90} \mathrm{P}_{60} \mathrm{~K}_{90}$ & $2.35^{\mathrm{cA}}$ & $1.94^{\mathrm{cB}}$ & $1.67^{\mathrm{cC}}$ & $1.39^{\mathrm{cD}}$ & \\
\hline
\end{tabular}

Same capital letter in a row and small letter in a column have no significant difference as per Duncan's Multiple Range Test.

Similar findings were also recorded by Thomas et al., (2002), Kamal and Yousuf (2012), Zewide et al., (2012), Asieku et al., (2015) Iwuagwu et al., (2016) and Misgina N. A. (2016).

\section{Gross return}

The organic and inorganic nutrients had significant simple and interaction effect on gross return of tikhur (Tables 1 and 2). The gross return ranges from 307444 to 587532 Rs.ha ${ }^{-1}$ and 382903 to 540879Rs.ha ${ }^{-1}$ with highest value due to application of fertilizer @ $\mathrm{N}_{90} \mathrm{P}_{60} \mathrm{~K}_{90} \mathrm{~kg} \mathrm{ha}^{-1}$ and vermicompost @ 30t $\mathrm{ha}^{-1}$, respectively. The effect of fertilizer @ $\mathrm{N}_{90} \mathrm{P}_{60} \mathrm{~K}_{90} \mathrm{~kg}$ ha $^{-1}$ was significantly higher than $\mathrm{N}_{0} \mathrm{P}_{0} \mathrm{~K}_{0}$ and $\mathrm{N}_{30} \mathrm{P}_{20} \mathrm{~K}_{30}$ but at par with $\mathrm{N}_{60} \mathrm{P}_{40} \mathrm{~K}_{60}$, similarly vermicompost @ 30t ha ${ }^{-}$ ${ }^{1}$ had significantly increased gross return as compared to 0 and $10 \mathrm{t} \mathrm{ha}^{-1}$ but its effect was at par as compared to vermicompost dose @ $20 \mathrm{t}$ $\mathrm{ha}^{-1}$.

In case of interaction effect (Table 2), the gross return of tikhur increased under all the 
fertilizer levels as we increased vermicompost levels up to $20 \mathrm{t} \mathrm{ha}^{-1}$, increasing vermicompost levels their after, did not increase gross return significantly. Similarly, the gross return of tikhur increased under all the vermicompost levels, as we increased fertilizer levels up to $\mathrm{N}_{60} \mathrm{P}_{40} \mathrm{~K}_{60}$, increasing fertilizer level further did not statistically increase the gross return. The higher gross return 639443Rs.ha ${ }^{-1}$ was achieved due to interaction of vermicompost @30 t ha ${ }^{-1}$ with fertilizer @ $\mathrm{N}_{90} \mathrm{P}_{60} \mathrm{~K}_{90} \mathrm{~kg} \mathrm{ha}^{-1}$ which was statistically at par with the interactions of $\mathrm{N}_{60} \mathrm{P}_{40} \mathrm{~K}_{60}$ X vermicompost @ 30t ha ${ }^{-1}$, $\mathrm{N}_{90} \mathrm{P}_{60} \mathrm{~K}_{90} X$ vermicompost @ 20t ha ${ }^{-1}$ and $\mathrm{N}_{60} \mathrm{P}_{40} \mathrm{~K}_{60} X$ vermicompost @ 20t ha ${ }^{-1}$. The combination of $\mathrm{N}_{60} \mathrm{P}_{40} \mathrm{~K}_{60} \mathrm{X} \quad 20 \mathrm{t} \mathrm{ha}^{-1}$ vermicompost was found superior as it saves cost on fertilizer and vermicompost and provides gross return comparable to higher doses of fertilizer and vermicompost.

\section{Net return}

The data presented in (Tables 1 and 2) revealed that organic and inorganic nutrients had significant simple and interaction effect on net return of tikhur. The net return ranges from 158485 to 375110 Rs.ha $^{-1}$ and 260176 to 320623 Rs.ha $^{-1}$ with highest value due to application of fertilizer @ $\mathrm{N}_{90} \mathrm{P}_{60} \mathrm{~K}_{90} \mathrm{~kg} \mathrm{ha}^{-1}$ and vermicompost @ $20 \mathrm{t} \mathrm{ha}^{-1}$, respectively. The effect of fertilizer @ $\mathrm{N}_{90} \mathrm{P}_{60} \mathrm{~K}_{90} \mathrm{~kg} \mathrm{ha}^{-1}$ was significantly higher than $\mathrm{N}_{0} \mathrm{P}_{0} \mathrm{~K}_{0}$ and $\mathrm{N}_{30} \mathrm{P}_{20} \mathrm{~K}_{30}$ but at par with $\mathrm{N}_{60} \mathrm{P}_{40} \mathrm{~K}_{60}$ and the application of vermicompost @ $20 \mathrm{tha}^{-1}$ had significantly higher net return as compared to 0,10 and $30 \mathrm{tha}^{-1}$.

In case of interaction effect(Table 2), the net return of tikhur increased under all the fertilizer levels as we increased vermicompost levels up to $20 \mathrm{t} \mathrm{ha}^{-1}$ increasing vermicompost levels their after, decreased net return significantly. Similarly, the net return of tikhur increased under all the vermicompost levels as we increased fertilizer levels up to $\mathrm{N}_{60} \mathrm{P}_{40} \mathrm{~K}_{60}$, increasing fertilizer level further did not increase the net return. The higher net return 397831.3Rs.ha ${ }^{-1}$ was achieved due to interaction of $\mathrm{N}_{60} \mathrm{P}_{40} \mathrm{~K}_{60} \mathrm{X} \quad 20 \mathrm{t} \quad \mathrm{ha}^{-1}$ vermicompost which was statistically at par with $\mathrm{N}_{90} \mathrm{P}_{60} \mathrm{~K}_{90} \mathrm{X} 20 \mathrm{t} \mathrm{ha}^{-1}$ vermicompost. The combination of $\mathrm{N}_{60} \mathrm{P}_{40} \mathrm{~K}_{60} \mathrm{X} \quad 20 \mathrm{t} \mathrm{ha}^{-1}$ vermicompost was superior as it gave the highest net return.

\section{Gross benefit: cost ratio}

The data presented in (Table 1 and 2) revealed that organic and inorganic nutrients had significant simple and interaction effect on gross $\mathrm{B}$ : $\mathrm{C}$ ratio. The gross $\mathrm{B}: \mathrm{C}$ ratio ranges from 2.13 to $2.86 \mathrm{Rs}$. $\mathrm{Re}^{-1}$ and 2.19 to 3.04 Rs. $\mathrm{Re}^{-1}$ (Table 1) with highest value due to application of fertilizer @ $\mathrm{N}_{60} \mathrm{P}_{40} \mathrm{~K}_{60} \mathrm{~kg} \mathrm{ha}^{-1}$ and vermicompost@0 0 ha $^{-1}$ respectively. The effect of fertilizer @ $\mathrm{N}_{60} \mathrm{P}_{40} \mathrm{~K}_{60} \mathrm{~kg} \mathrm{ha}^{-1}$ on gross $\mathrm{B}$ : $\mathrm{C}$ ratio was significantly higher than $\mathrm{N}_{0} \mathrm{P}_{0} \mathrm{~K}_{0}$ and $\mathrm{N}_{30} \mathrm{P}_{20} \mathrm{~K}_{30}$ but at par with $\mathrm{N}_{90} \mathrm{P}_{60} \mathrm{~K}_{90}$. Similarly vermicompost @ $0 \mathrm{t} \mathrm{ha}^{-1}$ had recorded significantly higher gross B: C ratio as compare to 20 and $30 \mathrm{tha}^{-1}$ but it was at par as compared to vermicompost dose @ $10 \mathrm{tha}^{-1}$.

In case of interaction effect (Table 2), the gross B: C ratio of tikhur decreased under all the fertilizer levels as we increased vermicompost levels up to $30 \mathrm{t} \mathrm{ha}^{-1}$. The gross $\mathrm{B}: \mathrm{C}$ ratio of tikhur increased significantly under all the vermicompost levels as we increased fertilizer levels up to $\mathrm{N}_{60} \mathrm{P}_{40} \mathrm{~K}_{60}$, increasing fertilizer levels further did not statistically increases the gross $\mathrm{B}: \mathrm{C}$ ratio. The higher gross $\mathrm{B}$ : $\mathrm{C}$ ratio 3.37 was achieved due to $\mathrm{N}_{60} \mathrm{P}_{40} \mathrm{~K}_{60} \mathrm{~kg} \mathrm{ha}^{-1} \mathrm{X} 0 \mathrm{t} \mathrm{ha}^{-1}$ vermicompost which was statistically at par with $\mathrm{N}_{90} \mathrm{P}_{60} \mathrm{~K}_{90} \mathrm{X}$ $0 \mathrm{t} \mathrm{ha}{ }^{-1}$ vermicompost. The combination of $\mathrm{N}_{60} \mathrm{P}_{40} \mathrm{~K}_{60} \mathrm{X} 0 \mathrm{t}$ ha ${ }^{-1}$ vermicompost was found superior due to no use of costlier input of vermicompost. 


\section{Net benefit: cost ratio}

The data presented in (Tables 1 and 2) revealed that the organic and inorganic nutrients had significant simple and interaction effect on net $\mathrm{B}$ : $\mathrm{C}$ ratio. The net $\mathrm{B}: \mathrm{C}$ ratio ranged from1.14 to $1.86 \mathrm{Rs} \cdot \mathrm{Re}^{-1}$ and 1.19 to 2.04 Rs.Re ${ }^{-1}$ (Table 1) with highest value due to application of fertilizer @ $\mathrm{N}_{60} \mathrm{P}_{40} \mathrm{~K}_{60} \mathrm{~kg} \mathrm{ha}^{-1}$ and vermicompost @ $0 \mathrm{tha}^{-1}$ respectively. The effect of fertilizer @ $\mathrm{N}_{60} \mathrm{P}_{40} \mathrm{~K}_{60} \mathrm{~kg} \mathrm{ha}^{-1}$ was significantly higher than $\mathrm{N}_{0} \mathrm{P}_{0} \mathrm{~K}_{0}$ and $\mathrm{N}_{30} \mathrm{P}_{20} \mathrm{~K}_{30}$ but at par with $\mathrm{N}_{90} \mathrm{P}_{60} \mathrm{~K}_{90}$. Contrarily, vermicompost @ $0 \mathrm{t}$ $\mathrm{ha}^{-1}$ had significantly higher net $\mathrm{B}$ : C ratio as compared to 20 and $30 \mathrm{t} \mathrm{ha}^{-1}$ but its effects was at par as compared to vermicompost @ $10 \mathrm{tha}^{-1}$.

In case of interaction effect (Table 2), the net B: C ratio of tikhur decreased under all the fertilizer levels as we increased vermicompost levels up to $30 \mathrm{tha}^{-1}$. The net $\mathrm{B}: \mathrm{C}$ ratio of tikhur increased under all vermicompost levels as we increased fertilizer levels up to $\mathrm{N}_{60} \mathrm{P}_{40} \mathrm{~K}_{60}$, increasing fertilizer levels further did not statistically increase the net $\mathrm{B}: \mathrm{C}$ ratio. The higher net $\mathrm{B}$ : $\mathrm{C}$ ratio 2.37 was achieved due to $\mathrm{N}_{60} \mathrm{P}_{40} \mathrm{~K}_{60} \mathrm{~kg} \quad \mathrm{ha}^{-1} \mathrm{X} \quad 0 \quad \mathrm{t} \quad \mathrm{ha}^{-1}$ vermicompost which was statistically at par with $\mathrm{N}_{90} \mathrm{P}_{60} \mathrm{~K}_{90} \mathrm{X} 0$ t ha ${ }^{-1}$ vermicompost. The combination of $\mathrm{N}_{60} \mathrm{P}_{40} \mathrm{~K}_{60} \quad \mathrm{X} \quad 0 \quad \mathrm{t} \quad \mathrm{ha}^{-1}$ vermicompost was found superior due to no use of costlier input of vermicompost. Similar findings were also recorded by Choudhary and Kumar (2013), Singh et al., (2016).

\section{References}

Amanullah, M. M., Vaiyapuri, K., Sathyamoorthi, K., Pazhanivelan, S. and Alageson A. 2007. Nutrient uptake, tuber yield of cassava (Mohinotesculenta) and soil fertility as influence by organic manure. J. Agron., 6(1): 183-187.

Asieku, Y., Otoo, E. and Asare, E. 2015. Yield and Storage Characteristics of White Yam
(Dioscorea rotundata Poir) as Influenced by Fertilizer Application and Time of Harvesting in Forest Zone of Ghana. Journal of Scientific Research \& Reports, 8(6): 1-7.

Choudhary, V. K., and Kumar, P. S. 2013. Maize production, economics and soil productivity under different organic source of nutrients in eastern Himalayan region, India. International Journal of Plant Production, 7(2): 167-186.

Ezeocha, V. C., Nwogha, J. S., Ohuoba, A. N. and Chunku, L. I. 2014. Evaluation of Poultry Manure Application Rates on the Nutrient Composition of Dioscorea bulbifera (Aerial yam).Nigerian Food Journal, 32(2): 92-96.

Hota, R., Jena, A. K, and Narayana, K. L. 2014. Effect of Inorganic and Organic Amendments on Yield of Cocoyam (Colocasia esculenta), and on Soil Properties. World Journal of Agricultural Research, 2(2): 70-81.

Iwuagwu, M. O., Okpara, D. A., Muoneke, C. O. and Ukaoma, A. A. 2016. Organic and mineral fertilizer effects on growth and yield of cocoyam in a tropical Ultisol in South Eastern Nigeria. J. Plant Breed. Crop Sci., 8(10): 175-181.

Kamal, M. Z. U., and Yousuf, M. N. 2012. Effect of Organic Manures on Growth, Rhizome Yield and Quality Attributes of Turmeric (Curcuma longa L.).The Agriculturists, 10 (1): 16-22.

Maheswarappa, H. P., Nanjappa, H. V., Hegde, M.R. and Biddappa, C. C. 2000. Nutrient content and uptake of arrowroot (Maranta arundinacea) as influenced by agronomic practices when grown as an intercrop in coconut (Cocos nucifera) garden. Indian J. Agron., 45:86-91.

Mama, A., Jeylan, J. and Aseffa A. W. 2016. Effects of different rates of organic and inorganic fertilizer on growth and yield components of potato (Solanum tuberosum 1.) in jimma are, south west Ethiopia. International Journal of Research, 4(11): 115-121.

Misgina, N. A., 2016. Effect of Phosphorus and Potassium Fertilizer Rates on Yield and Yield Component of Potato (Solanum tubersum L,) at K/Awlaelo, Tigray, 
Ethiopia. Food Science and Quality Management, 48:60-69.

Misra, S. H., and Dixit, V. K. 1983. Pharmaceutical studies on starches of some Zingiberaceous rhizomes. Indian Journal of Pharmaceutical Sciences, 45 (5): 216-220.

Odedina, S. A., Odedina, J. N., Ojeniyi, S. O. and Akinlana, F. 2012. Effect of Different Organic Nutrient Sources and Two NPK Rates on the Performance and Nutrient Contents of A Newly Released Cassava Variety. Journal of Life Sciences, 6: 10031007.

Ogbomo, K. E. L., and Remison, S. U. 2008. Growth and yield of white guinea yam (Dioscorea rotundata Poir.) influenced by NPK fertilization on a forest site in Nigeria. Journal of Tropical Agriculture, 46(1-2): 21-24.

Ramesan, K.K., Ahmed, P. and Oommen, M. 1996. Management of major plant nutrients for producing quality rhizomes in arrowroot. In: Proceedings of the 8th Kerala Science Congress Kerala State Council for Science, Technology and Environment, Government of Kerala, Kochi, 27-29: 133-134.

Sandhu, A. S., Sharma, S. P., Bhutani, R. D. and Khurana, S. C. 2014. Effects of planting date and fertilizer dose on plant growth attributes and nutrient uptake of potato (Solanum tuberosum L.). International Journal of Agricultural Sciences,4(5): 196202.

Singh, G., Choudhary, P., Sharma, H. L., Rawar, R. S. and Jat, B. L. 2016. Effect of Different Sources of Manuring on Growth, Yield and Quality of Capsicum (Capsicum annum) cv. California wonder under Low Cost Poly-House Condition. International Journal for Research in Applied Sciences \& Engineering Technology, 4: 530-552.

Suja, G., Nayar, T.V.R. and Ravindran, C.S. 2006. Influence of nutrient management in arrowroot (Maranta arundinacea L.) on biomass production, nutrient uptake and soil nutrient status. J. Root Crops, 32:162165.

Swadija, O. K., Padmanabhan, V. B. and Kumar, V. R. 2013. Growth and Yield of Arrowroot Intercropped in Coconut Garden as Influenced by Organic Management. Journal of Root Crops,39 (1): 67-72.

Thomas, A., Barche, S. and Singh, D. B. 2002. Influence of different levels of nitrogen and potassium on growth and yield of turmeric (Curcuma longa L.). Journal of spices and aromatic crops, 11(1): 74-77.

Veena, V., 2000. Integrated nutrient management for arrowroot (Maranta arundinacea L.) under partial shade. M.Sc. (Ag.) Thesis, Kerala Agricultural University, 107p.

Verma, V. K., Jha, A. K., Wanshnong, K. K. and Swer, T. L. 2012. Effect of Integrated Nutrient Management Modules on Growth, Yield and Quality Attributes in Taro (Colocasia esculenta L. Schott).Indian Journal of Hill Farming, 25(1): 21-25.

Zewide, I., Mohammed, A. and Tulu, S. 2012. Effect of Different Rates of Nitrogen and Phosphorus on Yield and Yield Components of Potato (Solanum tuberosum L.) at Masha District, South western Ethiopia. International Journal of Soil Science, 7(4): 146-15.

\section{How to cite this article:}

Karuna Kant Joshi, Girijesh Kumar Sharma, Tejpal Chandrakar and Deo Shankar. 2017. Influence of Graded Doses of Organic and Inorganic Nutrients on Growth, Yield and Economics of Tikhur (Curcuma aungustifolia Roxb.) in Inceptisol of Bastar Plateau. Int.J.Curr.Microbiol.App.Sci. 6(10): 1269-1277. doi: https://doi.org/10.20546/ijcmas.2017.610.151 